\title{
Secant Conditions in Power Network Stability
}

\author{
Nima Monshizadeh
}

\author{
Ioannis Lestas
}

\begin{abstract}
The problem of decentralized frequency control in power networks has received an increasing attention in recent years due to its significance in modern power systems and smart grids. Nevertheless, generation dynamics often involve turbine/governor dynamics, which, in conjunction with nonlinearities associated with generation and power flow, increase significantly the complexity in the analysis, and are not adequately addressed in the literature. In this paper we show how incremental secant gain conditions can be used in this context to reduce the conservatism in the analysis. The stability conditions derived are decentralized and are demonstrated throughout the paper with various examples.
\end{abstract}

\section{INTRODUCTION}

Due to the large scale penetration of renewable energy sources in the power grid, there has been an increasing interest in recent years in decentralized and distributed frequency control schemes in power networks. As a result of the nonlinearities associated with power flows, and also potential nonlinearites in the generation dynamics, a Lyapunov analysis is a natural tool often used in this context for stability analysis, e.g. the use of energy functions in [1]-[3], and more recent Lyapunov approaches in [4]-[8]. Extensions to differential algebraic models can be found in [9]-[11], see also [12].

Nevertheless, a feature that can complicate significantly such a Lyapunov analysis is the presence of turbine/governor dynamics in conjunction with nonlinearities often present in the generation or controllable demand side, such as deadbands and saturation. Such dynamics are often not explicitly addressed in the literature and various notable exceptions either resort to linearizations or propose gain conditions relative to the system damping that are lower than those encountered in practical implementations [13], [14]. In [15] a generalized passivity property on the aggregate bus dynamics was proposed, with further generalizations provided in [16], as a means of reducing the conservatism in the analysis. Systematic methods exist for verifying these properties for the case of linear systems. However, in the case of nonlinear systems, the problem of determining the minimum damping needed to passivate it is in general a nontrivial problem, as the form of the underlying storage function is unkown. Furthermore, simpler approaches that achieve passivation via a restricted $\mathcal{L}_{2}$ gain, can in general be restrictive, hence alternative methodologies need to be investigated.

Our aim in this paper is to show that the use of suitable incremental secant conditions, inspired by [17], [18], can

Nima Monshizadeh and Ioannis Lestas are with the Department of Engineering, University of Cambridge, Trumpington Street, Cambridge, CB2 1PZ, United Kingdom; e-mails: n.monshizadeh@eng.cam.ac.uk, icl20@ cam.ac.uk. The work was supported by ERC starting grant 679774 . facilitate the construction of classes of Lyapunov functions in this context and lead to stability conditions with reduced conservatism, in cases where a linearizaton is not appropriate. These conditions are decentralized and result in asymptotic stability for a range of equilibria of the system, and thus are able to cope with the uncertainties in load parameters and generation setpoints. The applicability of these conditions is demonstrated by means of several examples, illustrating that they provide stability guarantees with larger control gains, which in turn will enhance the performance of the network.

The structure of the paper is as follows. The power network model is provided in Section II. The desired asymptotic behavior of the system is characterized in Section III. The main results of the paper are provided in Section IV, and several exmaples are discussed to illustrate the applicability of the proposed stability conditions. The paper closes with conclusions in Section V. The proofs are omitted due to lack of space.

Notation. The $n \times n$ identity matrix is denoted by $I_{n}$, and $\mathbb{1}_{n}$ is the vector of all ones in $\mathbb{R}^{n}$, where the subscript is dropped if no confusion may arise. For $i \in\{1,2, \ldots, n\}$, by $\operatorname{col}\left(a_{i}\right)$ we denote the vector $\left(a_{1}, a_{2}, \ldots, a_{n}\right)$. For given vectors $a \in$ $\mathbb{R}^{n}$ and $b \in \mathbb{R}^{m}$, we denote the vector $\left(a^{T}, b^{T}\right)^{T} \in \mathbb{R}^{n+m}$ by $\operatorname{col}(a, b)$ or sometimes simply by $(a, b)$. Given a map $H: \mathbb{R}^{n} \rightarrow \mathbb{R}$, its transposed gradient is denoted by $\nabla H:=$ $\left(\frac{\partial H}{\partial x}\right)^{T}$

\section{Differential-Algebraic Model of POWer NETWORK}

We consider a structure-preserving model of power networks composed of load and generation buses. The topology of the grid is represented by a connected and undirected graph $\mathcal{G}(\mathcal{V}, \mathcal{E})$ with a vertex set (or buses) $\mathcal{V}=\{0,1, \ldots, n\}$, and an edge set $\mathcal{E}$ given by the set of unordered pairs $\{i, j\}$ of distinct vertices $i$ and $j$. The cardinality of $\mathcal{E}$ is denoted by $m$. We assume that the line admittances are purely inductive, and two nodes $\{i, j\} \in \mathcal{E}$ are connected by a nonzero real susceptance $\beta_{i j}<0$. The set of neighbors of the $i^{\text {th }}$ node is denoted by $\mathcal{N}_{i}=\{j \in \mathcal{V} \mid\{i, j\} \in \mathcal{E}\}$. The voltage phase angle at node $i \in \mathcal{V}$ is denoted by $\theta_{i} \in \mathbb{R}$. Voltage magnitudes $V_{i} \in \mathbb{R}^{+}$are assumed to be constant.

The set of generators is given by $\mathcal{V}_{g}=\left\{0,1, \cdots, n_{g}\right\}$. For each generator $i \in \mathcal{V}_{g}$, the phase $\theta_{i}$ evolves according to [19]

$$
\begin{aligned}
\dot{\theta}_{i} & =\omega_{i} \\
M_{i} \dot{\omega}_{i} & =-D_{i} \omega_{i}-p_{i}(\theta)+p_{i}^{*}+u_{i},
\end{aligned}
$$


where

$$
p_{i}(\theta)=\sum_{j \in \mathcal{N}_{i}}\left|\beta_{i j}\right| V_{i} V_{j} \sin \left(\theta_{i}-\theta_{j}\right)
$$

is the active power drawn from bus $i$. Here, $\omega_{i}$ is the frequency deviation from the nominal frequency (namely $50 \mathrm{~Hz}), M_{i}>0$ is the inertia constant, $D_{i}>0$ is the damping constant, the constant $p_{i}^{*}$ is the active power setpoint, and $u_{i} \in \mathbb{R}$ is the additional local power generation at bus $i$. The constant $p_{i}^{*}$ may also capture the constant power loads collocated with the $i$ th generator bus.

As for the loads, we consider constant power loads given by algebraic equations

$$
0=p_{i}^{*}-p_{i}(\theta)
$$

for each $i \in \mathcal{V}_{\ell}=\mathcal{V} \backslash \mathcal{V}_{g}$, where $p_{i}(\theta)$ is given by (2) and $p_{i}^{*}$ is constant. Note that constant impedance loads behave similarly to constant power loads if the voltages are approximately constant. We remark that the exact value of $p_{i}^{*}, i \in \mathcal{V}$, is not known a priori.

To capture a broad class of generation dynamics, let $u_{i} \in$ $\mathbb{R}$ be given by a nonlinear system of the form

$$
\begin{aligned}
\dot{\xi}_{i} & =f_{i}\left(\xi_{i},-\omega_{i}\right) \\
u_{i} & =h_{i}\left(\xi_{i},-\omega_{i}\right)
\end{aligned}
$$

where $f_{i}: \mathbb{R}^{n_{i}} \times \mathbb{R} \rightarrow \mathbb{R}^{n_{i}}$ and $h_{i}: \mathbb{R}^{n_{i}} \times \mathbb{R} \rightarrow \mathbb{R}$ are continuous and locally lipschitz. We sometimes denote such dynamical systems by $\Sigma_{i}\left(-\omega_{i}, \xi_{i}, u_{i}\right)$ in short. For any constant input $\omega_{i}=\bar{\omega}_{i}$, we assume that (4) possesses an isolated equilibrium $\xi_{i}=\bar{\xi}_{i}$, and we write $\bar{u}_{i}=h_{i}\left(\bar{\xi}_{i},-\bar{\omega}_{i}\right)$. We also assume that such an equilibrium is observable from the constant input-output pair $\left(-\bar{\omega}_{i}, \bar{u}_{i}\right)$, i.e, $\dot{\xi}_{i}=f_{i}\left(\xi_{i},-\bar{\omega}_{i}\right)$ together with $h_{i}\left(\xi_{i},-\bar{\omega}_{i}\right)=h_{i}\left(\bar{\xi}_{i},-\bar{\omega}_{i}\right)$ implies that $\xi_{i}=\bar{\xi}_{i}$.

Note that the dynamics (4) may include primary control, controllable loads, turbine governor dynamics, and possible static nonlinearities in the generation dynamics.

The power network dynamics can be written in vector form as the following differential algebraic system:

$$
\begin{aligned}
\dot{\theta}_{g} & =\omega_{g} \\
M \dot{\omega}_{g} & =-D \omega_{g}-p_{g}(\theta)+p_{g}^{*}+h\left(\xi,-\omega_{g}\right) \\
\dot{\xi} & =f\left(\xi,-\omega_{g}\right) \\
0 & =-p_{\ell}(\theta)+p_{\ell}^{*}
\end{aligned}
$$

where $M=\operatorname{blockdiag}\left(M_{i}\right), D=\operatorname{blockdiag}\left(D_{i}\right), \theta_{g}=$ $\operatorname{col}\left(\theta_{i}\right), \omega_{g}=\operatorname{col}\left(\omega_{i}\right), p_{g}(\theta)=\operatorname{col}\left(p_{i}(\theta)\right), p_{g}^{*}=\operatorname{col}\left(p_{i}\right)$, $\xi=\operatorname{col}\left(\xi_{i}\right), f=\operatorname{col}\left(f_{i}\right)$, and $h=\operatorname{col}\left(h_{i}\right)$ for $i \in \mathcal{V}_{g}$. Similarly, $p_{\ell}(\theta)=\operatorname{col}\left(p_{i}(\theta)\right)$ and $p_{\ell}^{*}=\operatorname{col}\left(p_{i}\right), i \in \mathcal{V}_{\ell}$.

Let $R$ be the incidence matrix of the graph. Note that, by associating an arbitrary orientation to the edges, the incidence matrix $R \in \mathbb{R}^{(n+1) \times m}$ is defined element-wise as $R_{i k}=1$, if node $i$ is the sink of the edge $k, d_{i k}=-1$, if $i$ is the source of the edge $k$, and $R_{i k}=0$ otherwise. In addition, let $\Gamma:=\operatorname{diag}\left(\gamma_{k}\right), \gamma_{k}=\left|\beta_{i j}\right| V_{i} V_{j}$, for each edge $k \sim\{i, j\}$ of the graph, where the edge numbering is in agreement with the incidence matrix $R$. Then the vector of active power transfer $p(\theta)=\operatorname{col}\left(p_{g}(\theta), p_{\ell}(\theta)\right)$ is written as

$$
p(\theta)=R \Gamma \sin \left(R^{T} \theta\right)=\left[\begin{array}{l}
R_{g} \\
R_{\ell}
\end{array}\right] \Gamma \sin \left(R^{T} \theta\right),
$$

where $R_{g}$ and $R_{\ell}$ are the submatrices of $R$ obtained by collecting the rows of $R$ indexed by $\mathcal{V}_{g}$ and $\mathcal{V}_{\ell}$, respectively. The operator $\sin (\cdot)$ is interpreted elementwise.

\section{SYNCHRONOUS SOLUTION AND A CHANGE OF COORDINATES}

We are interested in a synchronous motion of the power network, where the voltage phasors rotate with the same frequency. This writes as $\bar{\theta}_{i}(t)=\omega^{*} t+\bar{\theta}_{i}(0)$ for each $i \in \mathcal{V}$, with constant $\bar{\theta}_{i}(0) \in \mathbb{R}$. Note that a synchronous motion explicitly depends on time. In addition, note that if $\left(\theta, \omega_{g}, \xi\right)$ is a solution to (5), then $\left(\theta+c \mathbb{1}_{n+1}, \omega_{g}, \xi\right)$ is also a solution to (5) for any constant $c \in \mathbb{R}$. To get around this rotational invariance, we perform a change of coordinates by taking a phase angle of a generation bus, namely $\theta_{0}$, as a reference:

$$
\varphi_{i}=\theta_{i}-\theta_{0}, \quad i=1, \ldots, n .
$$

This new set of coordinates

$$
\left[\begin{array}{c}
0 \\
\varphi_{1} \\
\vdots \\
\varphi_{n}
\end{array}\right]=\left[\begin{array}{c}
\theta_{0} \\
\theta_{1} \\
\vdots \\
\theta_{n}
\end{array}\right]-\mathbb{1}_{n+1} \theta_{0} .
$$

Let $R_{\varphi} \in \mathbb{R}^{n \times m}$ denote the incidence matrix with its first row removed. Then, by the equality above and noting that $\mathbb{1} \in \operatorname{ker} R^{T}$, we have

$$
R^{T} \theta=R_{\varphi}^{T} \varphi
$$

Moreover, we have $\varphi=E^{T} \theta$ where $E^{T}=\left[\begin{array}{ll}-\mathbb{1}_{n} & I_{n}\end{array}\right]$. This can be rewritten as $\varphi=E_{g}^{T} \theta_{g}+E_{\ell}^{T} \theta_{\ell}$, where the matrix $E$ is partitioned accordingly as $E^{T}=\left[\begin{array}{ll}E_{g}^{T} & E_{\ell}^{T}\end{array}\right]$. Now, let $\varphi_{g}:=E_{g}^{T} \theta_{g}$ and $\varphi_{\ell}:=E_{\ell}^{T} \theta_{\ell}$. To clarify note that $\varphi, \varphi_{g}, \varphi_{\ell} \in \mathbb{R}^{n}$ and $\varphi=\varphi_{g}+\varphi_{\ell}$.

Then, the system (5) in the new coordinates reads as

$$
\begin{aligned}
\dot{\varphi}_{g} & =E_{g}^{T} \omega_{g} \\
M \dot{\omega}_{g} & =-D \omega_{g}-R_{g} \Gamma \sin \left(R_{\varphi}^{T} \varphi\right)+p_{g}^{*}+h\left(\xi,-\omega_{g}\right) \\
\dot{\xi} & =f\left(\xi,-\omega_{g}\right) \\
0 & =-R_{\ell} \Gamma \sin \left(R_{\varphi}^{T} \varphi\right)+p_{\ell}^{*}
\end{aligned}
$$

Let $U(\varphi):=-\mathbb{1}_{m}^{T} \Gamma \cos \left(R_{\varphi}^{T} \varphi\right)$, where again $\cos (\cdot)$ is defined elementwise. Clearly, $\nabla U(\varphi)=R_{\varphi} \Gamma \sin \left(R_{\varphi}^{T} \varphi\right)$. In addition, it is easy to see that $R=E R_{\varphi}$, and thus $R_{g}=E_{g} R_{\varphi}, R_{\ell}=E_{\ell} R_{\varphi}$. Then, (8) can be written as

$$
\begin{aligned}
\dot{\varphi}_{g} & =E_{g}^{T} \omega_{g} \\
M \dot{\omega}_{g} & =-D \omega_{g}-E_{g} \nabla U(\varphi)+p_{g}^{*}+h\left(\xi,-\omega_{g}\right) \\
\dot{\xi} & =f\left(\xi,-\omega_{g}\right) \\
0 & =-E_{\ell} \nabla U(\varphi)+p_{\ell}^{*}
\end{aligned}
$$


The representation above gives a differential algebraic model of the form

$$
\begin{aligned}
\dot{x} & =F(x, q) \\
0 & =g(x, q)
\end{aligned}
$$

where $x=\operatorname{col}\left(\varphi_{g}, \omega_{g}, \xi\right)$ and $q=\varphi_{\ell}$, noting that $\varphi=$ $\varphi_{g}+\varphi_{\ell}$. We assume that initial conditions are compatible with the algebraic equations, i.e, $0=g(x(0), q(0))$. For now, we also assume that the system above has a unique solution, for a nonzero interval of time, starting from any compatible initial condition. As will be observed later, this assumption is automatically satisfied since we will work in a region of state space where the algebraic constraints are regular, i.e, $\frac{\partial g}{\partial q}$ has full row rank.

As a result of this change of coordinate, a synchronous motion of the power network will be mapped to an equilibrium of the differential algebraic system (9), namely the point $\left(\bar{\varphi}, \bar{\omega}_{g}, \bar{\xi}\right)$ where $\bar{\omega}_{g}=\mathbb{1} \omega^{*}$ with $\omega^{*} \in \mathbb{R}$ being constant, and $\bar{\varphi} \in \mathbb{R}^{n}$ and $\bar{\xi} \in \mathbb{R}^{N}, N=\sum_{i \in \mathcal{V}_{g}} n_{i}$, are constant vectors satisfying

$$
\begin{aligned}
& 0=-D \mathbb{1} \omega^{*}-E_{g} \nabla U(\bar{\varphi})+p_{g}^{*}+h\left(\bar{\xi},-\mathbb{1} \omega^{*}\right), \\
& 0=-E_{\ell} \nabla U(\bar{\varphi})+p_{\ell}^{*} \\
& 0=f\left(\bar{\xi},-1 \omega^{*}\right) .
\end{aligned}
$$

We will refer to the equilibrium point $\left(\bar{\varphi}, \bar{\omega}_{g}, \bar{\xi}\right)$ as the synchronous solution of the power network. By (11), existence of such a solution imposes the following feasibility assumption:

Assumption 1: (Existence of a synchronous solution) There exists a constant $\omega^{*} \in \mathbb{R}$, a constant vector $\bar{\varphi} \in \mathbb{R}^{n}$ with $R_{\varphi}^{T} \bar{\varphi} \in\left(-\frac{\pi}{2}, \frac{\pi}{2}\right)^{m}$, and a constant vector $\bar{\xi} \in \mathbb{R}^{N}$ such that (11) is satisfied.

The additional requirement that $R_{\varphi}^{T} \bar{\varphi} \in\left(-\frac{\pi}{2}, \frac{\pi}{2}\right)^{m}$ means that the relative phase angles at steady-state should belong to the interval $\left(-\frac{\pi}{2}, \frac{\pi}{2}\right)$. The latter condition is often referred to as the security constraint and is ubiquitous in the literature, see e.g. [?], [5], [10], [20]. In case of linear generation dynamics, Assumption 1 can be verified using the results available on the solvability of (active) power flow equations, see e.g. [21].

\section{MAIN RESUlTS}

\section{A. Incremental passivity of the differential algebraic model}

Consider the differential algebraic system

$$
\begin{aligned}
\dot{\varphi}_{g} & =E_{g}^{T} \omega_{g} \\
M \dot{\omega}_{g} & =-D \omega_{g}-E_{g} \nabla U(\varphi)+p_{g}^{*}+u \\
0 & =-E_{\ell} \nabla U(\varphi)+p_{\ell}^{*} \\
y & =\omega_{g}
\end{aligned}
$$

with input-state-output $\left(u,\left(\varphi, \omega_{g}\right), \omega_{g}\right)$, where $u=\operatorname{col}\left(u_{i}\right)$. Clearly, (9) can be seen as a negative feedback interconnection of (12) with (4). As a first step towards a systematic stability analysis of (5), we identify an incremental passivity property of (12) with respect to a synchronous solution $\left(\bar{\varphi}, \bar{\omega}_{g}, \bar{\xi}\right)$. To formalise this property, we need the following definition:

Definition 1: Consider the differential algebraic system

$$
\begin{aligned}
\dot{x}_{o} & =f\left(x_{o}, x_{a}, u\right) \\
0 & =g\left(x_{o}, x_{a}\right) \\
y & =h\left(x_{o}, u\right)
\end{aligned}
$$

with input-state-output $(u, x, y)$, where $x=\operatorname{col}\left(x_{o}, x_{a}\right)$. System (13) is incrementally passive with respect to a point $(\bar{u}, \bar{x}, \bar{y}) \in \mathcal{U} \times \mathcal{X} \times \mathcal{Y}$, with $\bar{y}=h\left(\bar{x}_{0}, \bar{u}\right)$, if there exists a nonnegative ${ }^{1}$ and continuously differentiable function $S(x)$ and a positive semidefinite matrix $Q$, such that for all $x \in \mathcal{X}$ and $u \in \mathcal{U}$, the inequality

$$
\dot{S}(x) \leq-(y-\bar{y})^{T} Q(y-\bar{y})+(y-\bar{y})^{T}(u-\bar{u})
$$

holds. In case the matrix $Q$ is positive definite, we call the system output strictly incrementally passive with respect to $(\bar{u}, \bar{x}, \bar{y})$.

Now, we have the following proposition:

Proposition 1: Let $\left(\bar{\varphi}, \bar{\omega}_{g}\right)$, with $R_{\varphi}^{T} \bar{\varphi} \in\left(-\frac{\pi}{2}, \frac{\pi}{2}\right)^{n}$, be an equilibrium of (12) for some constant input $u=\bar{u}$, and let $\bar{y}=\bar{\omega}_{g}$. Then the differential algebraic system (12) is output strictly incrementally passive with respect to $\left(\bar{u},\left(\bar{\varphi}, \bar{\omega}_{g}\right), \bar{y}\right)$. In particular, the storage function $S$ given by

$$
\begin{aligned}
& S\left(\varphi, \omega_{g}\right)=\frac{1}{2}\left(\omega_{g}-\bar{\omega}_{g}\right)^{T} M\left(\omega_{g}-\bar{\omega}_{g}\right) \\
& +U(\varphi)-U(\bar{\varphi})-(\varphi-\bar{\varphi})^{T} \nabla U(\bar{\phi}),
\end{aligned}
$$

satisfies

$$
\dot{S}=-\left(\omega_{g}-\bar{\omega}_{g}\right)^{T} D\left(\omega_{g}-\bar{\omega}_{g}\right)+\left(\omega_{g}-\bar{\omega}_{g}\right)^{T}(u-\bar{u})
$$

Moreover, this storage function has a strict minimum at $\left(\bar{\varphi}, \bar{\omega}_{g}\right)$.

Recall that (9) is given by a negative feedback interconnection of (12) with (4). In case the generation dynamics (4) is (incrementally) passive as well, then by exploiting the result of Proposition 1, the closed-loop system enjoys suitable stability properties due to the well-known results on interconnection of passive systems, see Example 1. On the other hand, if (4) is not (incrementally) passive, then stability of closed-loop system is not automatically guaranteed, and it requires additional conditions.

Example 1: Suppose that the generation dynamics are given by static input-output relation $u_{i}=h_{i}\left(-\omega_{i}\right)$, where $h_{i}$ is strictly increasing for each $i \in \mathcal{V}_{g}$. Then, clearly, $-\left(\omega_{g}-\bar{\omega}_{g}\right)^{T}(u-\bar{u}) \geq 0$. Substituting this into (17) concludes stability of the equilibrium $\left(\bar{\varphi}, \bar{\omega}_{g}, \bar{\xi}\right)$, as $S$ has a strict minimum at $\left(\bar{\varphi}, \bar{\omega}_{g}\right)$ and $\dot{S}$ is nonpositive. Asymptotic stability follows by a suitable analysis of an invariant set of the system.

\footnotetext{
${ }^{1}$ Nonnegativity is assumed in $\mathcal{X}$. The set $\mathcal{X}$ can be shrunk as desired.
} 


\section{B. Small incremental-gain conditions}

Considering the nonlinearity of the generation dynamics, a first approach is to use an incremental $L_{2}$-gain argument. First, the following definition is needed:

Definition 2: (Incremental $L_{2}$ stablility) The system

$$
\begin{aligned}
\dot{x} & =f(x, u) \\
y & =h(x, u)
\end{aligned}
$$

with input-state-output $(u, x, y)$ is incrementally $L_{2}$ stable with respect to a point $(\bar{u}, \bar{x}, \bar{y}) \in \mathcal{U} \times \mathcal{X} \times \mathcal{Y}$, with $\bar{y}=h(\bar{x}, \bar{u})$, if there exists a nonnegative continuously differentiable function $S(x)$ and a scalar $\delta \in \mathbb{R}^{+}$such that for all $x \in \mathcal{X}$ and $u \in \mathcal{U}$, the inequality

$$
\dot{S}(x) \leq-\|y-\bar{y}\|^{2}+\delta^{2}\|u-\bar{u}\|^{2}
$$

holds. The system has an incremental $L_{2}$-gain not greater than $\delta$ in this case.

The notions of stability and asymptotic stability used here are those of [12]. Now, the following small incremental-gain result holds:

Proposition 2: Let Assumption 1 hold. Assume that (4) is incrementally $L_{2}$ stable with respect to $\left(-\bar{\omega}_{i}, \bar{\xi}_{i}, \bar{u}_{i}\right)$ and that the associated storage function has a strict minimum at this point. Let the incremental $L_{2}$-gain of (4) be not greater than $\delta_{i}$ for each $i \in \mathcal{V}_{g}$. Then, $\left(\bar{\varphi}, \bar{\omega}_{g}, \bar{\xi}\right)$ is an asymptotically stable equilibrium of (9) if, for each $i$,

$$
\delta_{i}<D_{i}
$$

Example 2: For each bus $i \in \mathcal{V}_{g}$, let $u_{i}$ be given by the nonlinear second-order dynamics

$$
\begin{aligned}
\tau_{\alpha, i} \dot{\alpha}_{i} & =-\nabla c_{i}\left(\alpha_{i}\right)+k_{i}\left(-\omega_{i}\right) \\
\tau_{\beta, i} \dot{\beta}_{i} & =-\beta_{i}+\alpha_{i} \\
u_{i} & =\beta_{i}
\end{aligned}
$$

where $\alpha_{i}, \beta_{i} \in \mathbb{R}$ are state variables, $\tau_{\alpha, i}, \tau_{\beta, i} \in \mathbb{R}^{+}$are time constants, and $c_{i}: \Omega_{c} \rightarrow \mathbb{R}$ is a strongly convex function, i.e. there exists $\rho_{i}^{c} \in \mathbb{R}^{+}$such that

$$
\left(\alpha_{i}-\bar{\alpha}_{i}\right)\left(\nabla c_{i}\left(\alpha_{i}\right)-\nabla c_{i}\left(\bar{\alpha}_{i}\right)\right) \geq \rho_{i}^{c}\left(\alpha_{i}-\bar{\alpha}_{i}\right)^{2},
$$

for all $\alpha, \bar{\alpha} \in \Omega_{c}$. In addition, the map $k_{i}: \Omega_{k} \rightarrow \mathbb{R}$ satisfies $\left|k_{i}\left(-\omega_{i}\right)-k_{i}\left(-\bar{\omega}_{i}\right)\right| \leq \rho_{i}^{k}\left|\omega_{i}-\bar{\omega}_{i}\right|, \forall \omega_{i}, \bar{\omega}_{i} \in \Omega_{k}$. Let $\left(\bar{\alpha}_{i}, \bar{\beta}_{i}\right)$ denote the equilibrium of (21) resulting from a constant input $-\bar{\omega}_{i}$. By defining $v_{i}=k_{i}\left(-\omega_{i}\right), \bar{v}_{i}=$ $k_{i}\left(-\bar{\omega}_{i}\right), \bar{u}_{i}=\bar{\beta}_{i}$, and choosing the storage function

$$
S_{i}=\frac{\tau_{\alpha, i}}{\rho_{i}^{c}}\left(\alpha_{i}-\bar{\alpha}_{i}\right)^{2}+\tau_{\beta, i}\left(\beta_{i}-\bar{\beta}_{i}\right)^{2},
$$

it is easy to see that

$\dot{S}_{i} \leq\left(\frac{1}{\rho_{i}^{c}}\right)^{2}\left(v_{i}-\bar{v}_{i}\right)^{2}-\left(\beta_{i}-\bar{\beta}_{i}\right)^{2} \leq\left(\frac{\rho_{i}^{k}}{\rho_{i}^{c}}\right)^{2}\left(\omega_{i}-\bar{\omega}_{i}\right)^{2}-\left(u_{i}-\bar{u}_{i}\right)^{2}$
Therefore the system (21) has an incremental $L_{2}$-gain $\leq \frac{\rho_{i}^{k}}{\rho_{i}^{c}}$, and by Proposition 2 , the equilibrium $(\bar{\varphi}, \bar{\omega}, \bar{\xi})$ with $\bar{\xi}_{i}=$ $\left(\bar{\alpha}_{i}, \bar{\beta}_{i}\right)$ is asymptotically stable if $\rho_{i}^{k}<\rho_{i}^{c} D_{i}$ for each $i \in$ $\mathcal{V}_{g}$. In the special case where $c_{i}=\frac{1}{2} \alpha_{i}^{2}, \nabla c_{i}$ becomes linear, and the conditions simplifies to $\rho_{i}^{k}<D_{i}$. This is consistent with the result obtained in [14] for a linearized power flow model without algebraic constraints.

\section{Incremental secant conditions}

While $L_{2}$-gain arguments are powerful and applicable to fairly general classes of nonlinear generation dynamics, they often lead to conservative conditions require a substantial amount of damping for stability guarantees. To put forward an alternative approach and obtain less conservative stability conditions, a key observation is that generation dynamics typically can be written as a cascaded interconnection of output-strictly incrementally passive systems. We impose this observation as an assumption, and will study its applicability on several examples later in the manuscript. Before providing the explicit assumption, note that we use the same (incremnetal) passivity notion as in Definition 1 for systems of ordinary differential equations as a special case. Moreover, we call a static input-output map $y=\phi(u)$ output strictly incrementally passive with respect to a point $(\bar{u}, \bar{y})$, with $\bar{y}=\phi(\bar{u})$, if (14) holds with $S=0$ and $Q>0$, i.e,

$$
0 \leq-(y-\bar{y})^{T} Q(y-\bar{y})+(y-\bar{y})^{T}(u-\bar{u}) .
$$

Assumption 2: For each $i \in \mathcal{V}_{g}$, the system (4) can be written as a cascaded interconnection of single-input singleoutput subsystems $\Sigma_{i j}, j \in \mathcal{P}=\left\{1, \ldots, n_{\mathcal{P}}\right\}$, such that

1) Each input-state-output block $\Sigma_{i j}\left(v_{i j}, \xi_{i j}, z_{i j}\right)$ is output strictly incrementally passive with respect to $\left(\bar{v}_{i j}, \bar{\xi}_{i j}, \bar{z}_{i j}\right)$, namely (14) holds for some storage function $S_{i j}$ and a positive scalar $Q_{i j}$. The storage function $S_{i j}$ has a strict minimum at $\left(\bar{v}_{i j}, \bar{\xi}_{i j}, \bar{z}_{i j}\right)$.

2) Each static block $\Sigma_{i j}$ given by input-output relation $z_{i j}=\phi_{i j}\left(v_{i j}\right)$ is output strictly incrementally passive with respect to $\left(\bar{v}_{i j}, \bar{z}_{i j}\right)$, namely (22) holds for some positive scalar $Q_{i j}$.

Here, $\omega_{i}=v_{i 1}, z_{i(k-1)}=v_{i k}$ for $k=2, \ldots, n_{\mathcal{P}}, z_{i n_{\mathcal{P}}}=u_{i}$, and $\operatorname{col}\left(\xi_{i j}\right), j \in \mathcal{P}$, is equal to the vector $\xi_{i}$ in (4), $i \in$ $\mathcal{V}_{g}$. The variables with the overlines are defined consistently, noting that $\bar{\xi}_{i}=\operatorname{col}\left(\operatorname{col}\left(\bar{\xi}_{i j}\right)\right)$ are such that (11) is satisfied.

Remark 1: For linear blocks, the incremental passivity property in Assumption 2 reduces to passivity. For static blocks, the incremental passivity property amounts to an incremental sector boundedness where the slope of nonlinearity does not exceed $Q_{i j} \in \mathbb{R}$.

Unlike parallel interconnections, cascaded interconnection does not preserve passivity properties, and hence closed loop stability is not automatically guaranteed. However, under Assumption 2, the shortage of (incremental) passivity can be quantified by adapting the so-called "secant conditions" [17], [18], to our incremental setting, where loads act as external constant disturbances to the system. This brings us to the following theorem: 
Theorem 1: Let Assumptions 1 and 2 hold. Then, $\left(\bar{\varphi}, \bar{\omega}_{g}, \bar{\xi}\right)$ is an asymptotically stable equilibrium of (9) if

$$
D_{i}^{-1}<Q_{i 1} \cdots Q_{i n_{\mathcal{P}}}\left(\sec \left(\frac{\pi}{n_{\mathcal{P}}+1}\right)\right)^{n_{\mathcal{P}}+1}
$$

for each $i \in \mathcal{V}_{g}$.

Example 3: Suppose that the generation dynamics at each bus $i \in \mathcal{V}_{g}$ is given by the first order model

$$
\begin{aligned}
\tau_{\xi, i} \dot{\xi}_{i} & =-\xi_{i}+k_{i}\left(-\omega_{i}\right) \\
u_{i} & =\xi_{i}
\end{aligned}
$$

with $\tau_{\xi, i} \in \mathbb{R}^{+}$. The map $k_{i}: \Omega_{k} \rightarrow \mathbb{R}$ is monotonically increasing and satisfies $\left|k_{i}\left(-\omega_{i}\right)-k_{i}\left(-\bar{\omega}_{i}\right)\right| \leq \rho_{i}\left|\omega_{i}-\bar{\omega}_{i}\right|$, $\forall \omega_{i}, \bar{\omega}_{i} \in \Omega_{k}$, for some $\rho_{i} \in \mathbb{R}^{+}$. Typical examples of $k_{i}$ include deadband nonlinearities and inverse of marginal costs in primary control [14], [22]. The first order dynamics can be obtained from [23, p.382] by neglecting the fast dynamics of the governor, see e.g. [24].

Clearly we have

$$
\begin{aligned}
0 \leq- & \frac{1}{\rho_{i}}\left|k_{i}\left(-\omega_{i}\right)-k_{i}\left(-\bar{\omega}_{i}\right)\right|^{2} \\
& -\left(\omega_{i}-\bar{\omega}_{i}\right)\left(k_{i}\left(-\omega_{i}\right)-k_{i}\left(-\bar{\omega}_{i}\right)\right) .
\end{aligned}
$$

Hence, $z_{i 1}:=k_{i}\left(-\omega_{i}\right)$ defines an incrementally passive map. Moreover, by taking the storage function $S_{i 2}=\frac{1}{2} \tau_{\xi, i}\left(\xi_{i}-\right.$ $\left.\bar{\xi}_{i}\right)^{2}$ with $\bar{\xi}_{i}$ denoting the equilibrium of (24) resulting from the constant input $-\bar{\omega}_{i}$, we have

$$
\dot{S}_{i 2}=-\left(\xi_{i}-\bar{\xi}_{i}\right)^{2}+\left(\xi_{i}-\bar{\xi}_{i}\right)\left(v_{i 2}-\bar{v}_{i 2}\right),
$$

where $v_{i 2}=k_{i}\left(-\omega_{i}\right)=z_{i 1}$ and $\bar{v}_{i 2}=k_{i}\left(-\bar{\omega}_{i}\right)$. This implies that the system with input-state-output $\left(v_{i 2}, \xi_{i}, u_{i}\right)$ is incrementally passive. Therefore, Assumption 2 is satisfied with $n_{\mathcal{P}}=2, Q_{i 1}=\rho_{i}^{-1}$ and $Q_{i 2}=1$. Consequently, by Theorem $1,(\bar{\varphi}, \bar{\omega}, \bar{\xi})$ is asymptotically stable if

$$
\rho_{i}<8 D_{i} .
$$

This condition is eight times less conservative than sufficient damping conditions obtained from $L_{2}$-gain arguments.

Example 4: Let the generation dynamics at each bus $i \in$ $\mathcal{V}_{g}$ be given by the nonlinear second-order dynamics in (21), see also [23, p.382], [14], [25]. We split the dynamics into three cascaded subdynamics, namely

$$
\begin{aligned}
& z_{i 1}=k_{i}\left(-\omega_{i}\right), \\
\tau_{\alpha, i} \dot{\alpha}_{i} & =-\nabla c_{i}\left(\alpha_{i}\right)+v_{i 2}, \quad v_{i 2}=z_{i 1}, \\
z_{i 2} & =\alpha_{i},
\end{aligned}
$$

and

$$
\begin{aligned}
\tau_{\beta, i} \dot{\beta}_{i} & =-\beta_{i}+v_{i 3}, \quad v_{i 3}=z_{i 2} \\
u_{i} & =\beta_{i} .
\end{aligned}
$$

As before, the first block (26) satisfies the incremental passivity property (25) with $\rho_{i}$ being replaced by $\rho_{i}^{k}$. The storage functions $S_{i 2}=\frac{1}{2}\left(\alpha_{i}-\bar{\alpha}_{i}\right)^{2}$ and $S_{i 3}=\frac{1}{2}\left(\beta_{i}-\bar{\beta}_{i}\right)^{2}$ yields the incremental passivity of the second and third subsystems, (27) and (28), with corresponding coefficients $Q_{i 2}=\rho_{i}^{c}$ and $Q_{i 3}=1$, respectively. Therefore, noting that $n_{\mathcal{P}}=3$, the secant condition in Theorem 1 reads as

$$
\frac{\rho_{i}^{k}}{\rho_{c}^{i}}<4 D_{i} .
$$

Again note that the condition above is 4 times less conservative than the one resulting from an $L_{2}$-gain argument, see Example 2.

Next, it is illustrative to consider the same dynamics as before but with an additional nonlinear map at the outputs, namely

$$
u_{i}=h_{i}\left(\beta_{i}\right),
$$

where $h_{i}: \Omega_{h} \rightarrow \mathbb{R}$ is strictly increasing and satisfies $\left|h_{i}\left(\beta_{i}\right)-h_{i}\left(\bar{\beta}_{i}\right)\right| \leq \rho_{i}^{h}\left|\beta_{i}-\bar{\beta}_{i}\right|, \forall \beta_{i}, \bar{\beta}_{i} \in \Omega_{h}$, for some $\rho_{i}^{h} \in$ $\mathbb{R}^{+}$. Hence, $h_{i}$ defines an incrementally passive map, and can be treated as a new block next to the three subsystems (26), (27), and (28a). Then, inequality (23) with $n_{\mathcal{P}}=4$ gives the stability condition $\frac{\rho_{i}^{h} \rho_{i}^{k}}{\rho_{c}^{i}}<2.88 D_{i}$. However, noting that the secant condition becomes more conservative as the number of cascaded subsystems increases, a compelling alternative is to possibly refine the storage function. To this end, let $S_{i 3}$ be redefined as

$$
S_{i 3}:=H_{i}\left(\beta_{i}\right)-H_{i}\left(\bar{\beta}_{i}\right)-\left.\left(\beta_{i}-\bar{\beta}_{i}\right) \frac{\partial H_{i}}{\partial \beta_{i}}\right|_{\beta_{i}=\bar{\beta}_{i}},
$$

where

$$
H_{i}\left(\beta_{i}\right)=\int_{\bar{\beta}_{i}}^{\beta_{i}} h_{i}\left(\tilde{\beta}_{i}\right) d \tilde{\beta}_{i} .
$$

We note that $S_{i 3}$ is associated with the Bregman distance defined on the function $H_{i}$ with respect to the point $\bar{\beta}_{i}$ [26]. Since $h_{i}$ is strictly increasing, the function $H_{i}$ is strictly convex, and therefore the storage function $S_{i 3}$ is positive definite. Computing the time derivative of $S_{i 3}$ along the solutions of (28a) yields

$$
\begin{aligned}
& \dot{S}_{i 3}=-\left(\beta_{i}-\bar{\beta}_{i}\right)\left(h_{i}\left(\beta_{i}\right)-h_{i}\left(\bar{\beta}_{i}\right)\right) \\
&+\left(h_{i}\left(\beta_{i}\right)-h_{i}\left(\bar{\beta}_{i}\right)\right)\left(v_{i 3}-\bar{v}_{i 3}\right) \\
& \leq-\frac{1}{\rho_{i}^{h}}\left(u_{i}-\bar{u}_{i}\right)^{2}+\left(u_{i}-\bar{u}_{i}\right)\left(v_{i 3}-\bar{v}_{i 3}\right) .
\end{aligned}
$$

This amounts to the incremental passivity property of (29) with $Q_{i 3}=\left(\rho_{i}^{h}\right)^{-1}$. Hence, Theorem 1 can be applied with $n_{\mathcal{P}}=3$, which gives the more relaxed stability condition

$$
\frac{\rho_{i}^{h} \rho_{i}^{k}}{\rho_{c}^{i}}<4 D_{i} .
$$

\section{CONCLUSiOnS}

We have provided a Lyapunov stability analysis of a differential algebraic model of frequency dynamics in power networks with turbine governor dynamics, static and dynamic nonlinearities. As observed this model has an incremental passivity property which can be leveraged in the stability analysis of the power network. As an immediate application, incremental $L_{2}$-gain conditions will guarantee stability of 
the overall closed loop dynamics. As confirmed by several examples, it turns out that such conditions require substantial amount of damping which may lead to an overly conservative design. To decrease this conservatism, we put forward incremental secant gain conditions which rely on suitable cascaded decomposition of the generation dynamics. These conditions are rather mild and are applicable to generation dynamics with multiple nonlinearities. Interesting directions for future research are to consider more accurate models including voltage control dynamics and secondary frequency control schemes, and to investigate robustness against measurement noise.

\section{REFERENCES}

[1] N. Tsolas, A. Arapostathis, and P. Varaiya, "A structure preserving energy function for power system transient stability analysis," IEEE Transactions on Circuits and Systems, vol. 32, no. 10, pp. 1041-1049, 1985.

[2] C.-C. Chu and H.-D. Chiang, "Constructing analytical energy functions for lossless network-reduction power system models: Framework and new developments," Circuits, systems, and signal processing, vol. 18, no. 1, pp. 1-16, 1999.

[3] H.-D. Chang, C.-C. Chu, and G. Cauley, "Direct stability analysis of electric power systems using energy functions: theory, applications, and perspective," Proceedings of the IEEE, vol. 83, no. 11, pp. 1497$1529,1995$.

[4] C. De Persis and N. Monshizadeh, "Bregman storage functions for microgrid control," IEEE Transactions on Automatic Control, vol. 63, no. 1 , pp. 53-68, 2018.

[5] S. Trip, M. Bürger, and C. De Persis, "An internal model approach to (optimal) frequency regulation in power grids with time-varying voltages," Automatica, vol. 64, pp. 240-253, 2016.

[6] C. Zhao, E. Mallada, and F. Dörfler, "Distributed frequency control for stability and economic dispatch in power networks," in American Control Conference (ACC), 2015. IEEE, 2015, pp. 2359-2364.

[7] B. Jayawardhana, R. Ortega, E. García-Canseco, and F. Castanos, "Passivity of nonlinear incremental systems: Application to pi stabilization of nonlinear RLC circuits," Systems \& control letters, vol. 56, no. 9, pp. 618-622, 2007.

[8] M. Arcak, C. Meissen, and A. Packard, Networks of Dissipative Systems: Compositional Certification of Stability, Performance, and Safety. Springer, 2016.

[9] J. Schiffer and F. Dörfler, "On stability of a distributed averaging pi frequency and active power controlled differential-algebraic power system model," in Control Conference (ECC), 2016 European. IEEE, 2016, pp. 1487-1492.

[10] N. Monshizadeh, C. De Persis, A. J. van der Schaft, and J. M. Scherpen, "A novel reduced model for electrical networks with constant power loads," IEEE Transactions on Automatic Control, 2017.

[11] C. De Persis, N. Monshizadeh, J. Schiffer, and F. Dörfler, "A Lyapunov approach to control of microgrids with a network-preserved differential-algebraic model," in Decision and Control (CDC), 2016 IEEE 55th Conference on. IEEE, 2016, pp. 2595-2600.

[12] D. Hill and I. Mareels, "Stability theory for differential/algebraic systems with application to power systems," Circuits and Systems, IEEE Transactions on, vol. 37, no. 11, pp. 1416-1423, 1990.

[13] S. Trip and C. De Persis, "Optimal generation in structure-preserving power networks with second-order turbine-governor dynamics," in Control Conference (ECC), 2016 European. IEEE, 2016, pp. 916921.

[14] C. Zhao and S. Low, "Optimal decentralized primary frequency control in power networks," in Decision and Control (CDC), 2014 IEEE 53rd Annual Conference on. IEEE, 2014, pp. 2467-2473.

[15] A. Kasis, E. Devane, C. Spanias, and I. Lestas, "Primary frequency regulation with load-side participationpart i: Stability and optimality," IEEE Transactions on Power Systems, vol. 32, no. 5, pp. 3505-3518, 2017.

[16] E. Devane, A. Kasis, M. Antoniou, and I. Lestas, "Primary frequency regulation with load-side participationpart ii: Beyond passivity approaches," IEEE Transactions on Power Systems, vol. 32, no. 5, pp. 3519-3528, 2017.
[17] E. D. Sontag, "Passivity gains and the secant condition for stability," Systems \& control letters, vol. 55, no. 3, pp. 177-183, 2006.

[18] M. Arcak and E. D. Sontag, "Diagonal stability of a class of cyclic systems and its connection with the secant criterion," Automatica, vol. 42, no. 9, pp. 1531-1537, 2006.

[19] P. Kundur, N. J. Balu, and M. G. Lauby, Power system stability and control. McGraw-hill New York, 1994, vol. 7.

[20] F. Dörfler, J. Simpson-Porco, and F. Bullo, "Breaking the hierarchy: Distributed control \& economic optimality in microgrids," arXiv preprint arXiv:1401.1767, 2014.

[21] F. Dörfler, M. Chertkov, and F. Bullo, "Synchronization in complex oscillator networks and smart grids," Proceedings of the National Academy of Sciences, vol. 110, no. 6, pp. 2005-2010, 2013.

[22] A. Kasis, E. Devane, and I. Lestas, "Primary frequency regulation in power networks with ancillary service from load-side participation," in Proceedings of the 20th IFAC World Congress IFAC, 2017.

[23] A. Bergen and V. Vittal, "Power system analysis,(2nd)," 1999.

[24] N. Li, C. Zhao, and L. Chen, "Connecting automatic generation control and economic dispatch from an optimization view," IEEE Transactions on Control of Network Systems, vol. 3, no. 3, pp. 254-264, 2016.

[25] M. Ourari, L. Dessaint, and V. Do, "Dynamic equivalent modeling of large power systems using structure preservation technique," Power Systems, IEEE Transactions on, vol. 21, no. 3, pp. 1284-1295, 2006.

[26] L. M. Bregman, "The relaxation method of finding the common point of convex sets and its application to the solution of problems in convex programming," USSR computational mathematics and mathematical physics, vol. 7, no. 3, pp. 200-217, 1967. 